\title{
On the Impact of Group Delay Variations on GNSS Time and Frequency Transfer
}

\author{
Tobias Kersten ${ }^{\star 1}$, Steffen Schön ${ }^{1,2}$ and Ulrich Weinbach ${ }^{1,2}$
}

\section{Contents}

\section{Introduction}

2 Concept of GDV Determination

2.1 Observation Model . . . . . . . . . . . . . . . . 2

2.2 Mathematical Model . . . . . . . . . . . . . . . 2

3 Discussion of Group Delay Variations (GDV)

4 Application to time transfer 4

4.1 Simulation Strategy . . . . . . . . . . . . . 4

4.2 PPP frequency transfer $\ldots \ldots \ldots \ldots \ldots$

5 Conclusion

Acknowledgements

References

\section{Introduction}

Global Navigation Satellite Systems (GNSS) are not only used for navigation and positioning but are also widely adopted by timing laboratories for international time and frequency transfer. In addition to the Two Way Satellite Time and Frequency Transfer (TWSTFT), three GNSS based techniques are in use: (1) GPS Common View (CV), (2) All-In-View, and recently (3) Precise Point Positioning (PPP), cf, e.g., [1, 2], or [3].

Using the GPS precise P code has two advantages. First, the noise level of the $\mathrm{P}$ code is considerably smaller than that one of the clear access (C/A) code. Second, the first order ionospheric delays can be eliminated forming the ionosphere free linear combination $\mathrm{P} 3$, since the $\mathrm{P}$ code is modulated on both frequencies as signal L1 P(Y) and L2 P(Y). Generally, lower signal to noise ration (SNR) at low elevation may show large variations [4] and furthermore the $\mathrm{P}$ code is modulated with the encrypted Y code. Therefore efforts have been made by manufacturers to develop different strategies for tracking this signal.

For time and frequency transfers, different error sources related to the receiver-antenna combination have to be considered, like e.g., hardware delays in the cable and receiver as well as the temperature sensitivity of the equipment. In this paper we will discuss the impact of the so called Group Delay Variations (GDV), i.e. delays that are dependent on the receiver antenna and the azimuth and elevation of the incoming signal. In literature, GDV have been addressed, e.g. in the context of GNSS based landing approaches [5], or satellite orbit determination [6]. But also time and frequency transfer methods can be affected, especially when combining the two orders of magnitude more precise carrier phase observables with code observables [7-9]. First experiments using the absolute antenna calibration unit to determine the elevation dependent variations of the code Group Delays were discussed in [10] for several antennas and receivers. Within these studies GDV for geodetic GNSS antenna with magnitudes in the order of $1 \mathrm{~m}(3.3 \mathrm{~ns})$ were obtained with an accuracy of about $5 \mathrm{~cm}(0.17 \mathrm{~ns})$.

The paper is organized as follows: In the first section the 
methodology to determine the GDV is explained. In section 3 the obtained GDV for several geodetic antennae are discussed and validated experimentally. In the last section we analyze the possible impact of the GDV on time and frequency transfer for European and transatlantic links using simulations and real data processed with a PPP approach.

\section{Concept of GDV Determination}

With the Hannover Concept of absolute antenna calibration a well-established experiment and calibration facility exists. Since 2000, Phase Centre Variations (PCV) for GPS and GLONASS carrier phases on L1/L2 are successfully and routinely determined as described in detail in, e.g., [11,12], or [13]. For this investigation, we made use of this technique and expanded the processing algorithms in our IfE GNSS software to use this approach to recover GDV with the actual available GNSS signals in the field. The facility as well as the concept of this approach are shown in Figure 1.

\subsection{Observation Model of Group Delay Variations (GDV)}

For accessing GDV at the observation level, we apply an approach similar to that one used for carrier phase observations as shown in [12] and [14]. The undifferenced code observation $P_{A}^{j}$ in meters from a satellite $j$ to a station $A$ reads:

$$
\begin{aligned}
P_{A}^{j}=\rho_{A}^{j} & +c\left(\delta t_{A}-\delta t^{j}\right)+T_{A}^{j}+I_{A}^{j}+d_{A}-d^{j} \\
& +R E L_{A}^{j}+M P_{A}^{j}+G D V(\alpha, e)+\varepsilon_{A}^{j},
\end{aligned}
$$

with the geometric distance $\rho_{A}^{j}$, the receiver and satellite clock error in meter $c \delta t_{A}, c \delta t^{j}$, the signal delay in the receiver $d_{A}$ and in the satellite $d^{j}$, the relativistic effect $R E L_{A}^{j}$, the multipath error $M P_{A}^{j}$, and code noise $\varepsilon_{A}^{j}$, as well as the tropospheric path delay $T_{A}^{j}$ and the ionospheric path delay $I_{A}^{j}$, [15]. In addition, we introduce the Group Delay Variations $\operatorname{GDV}(\alpha, e)$ to the code observables as a function of the elevation $e$ and azimuth $\alpha$ of the incident ray.

For the elimination of distance dependent effects as well as effects induced by the satellite clock, inter-station single differences $S D_{A B}^{j}=P_{A}^{j}-P_{B}^{j}$ are formed:

$$
\begin{array}{r}
S D_{A B}^{j}\left(t_{l}\right)=G D V_{A B}^{j}\left(\alpha, e, t_{l}\right)+M P_{A B}^{j}\left(t_{l}\right) \\
+c \delta t_{A B}\left(t_{l}\right)+\varepsilon_{A B}^{j}\left(t_{l}\right),
\end{array}
$$

with the differential Group Delay Variation $G D V_{A B}^{j}\left(\alpha, e, t_{l}\right)$, the effect of multipath at both stations $M P_{A B}^{j}\left(t_{l}\right)$, the differential receiver clock error $c \delta t_{A B}\left(t_{l}\right)$ in meters, as well as the code noise $\varepsilon_{A B}^{j}\left(t_{l}\right)$ with a magnitude of $\sqrt{2} \cdot \varepsilon^{j}\left(t_{l}\right)$. The observation epoch is denoted by $t_{l}$.

A Stanford Rubidium FS725 was used as common oscillator, thus the differential receiver clock error is constant. Its amount equals the initial clock offset between both receivers.

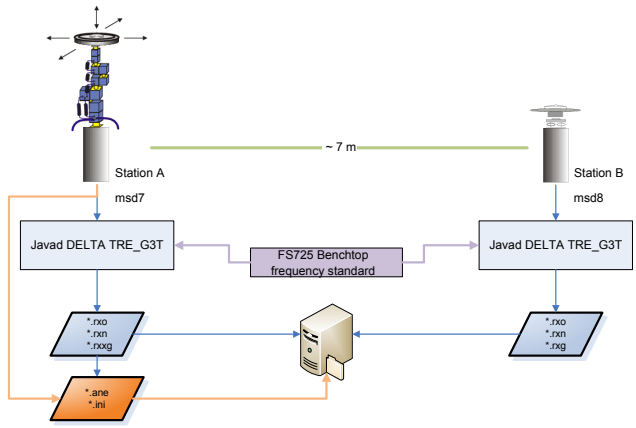

Figure 1. Calibration facility of the Hannover Concept for absolute antenna calibration at the Institut für Erdmessung, University of Hannover.

Simply using inter-station single differences, like in equation (2), would largely reduce the GDV as well. However, if the orientation of the antenna under test is changed in very precise and predictable steps between subsequent epochs, we eliminate the impact of the reference antenna and access the GDV of the test-antenna at epoch $t_{l}$ like

$$
\begin{aligned}
\Delta S D_{A, B}^{j}\left(t_{l}, t_{l-1}\right)= & S D_{A B}^{j}\left(t_{l-1}\right)-S D_{A B}^{j}\left(t_{l}\right) \\
= & \Delta S D V_{A}^{j}\left(t_{l-1}, t_{l}\right) \\
& +\Delta M P_{A B}\left(t_{l-1}, t_{l}\right) \\
& +\Delta \varepsilon_{A B}^{j}\left(t_{l}\right) .
\end{aligned}
$$

Due to a maximum offset of $5^{\mathrm{s}}$ between subsequent epochs, the multipath term at station $B$ is eliminated and largely reduced for the antenna under test mounted on the robot. Since both receivers are connected to the same oscillator (Stanford Rubidium FS725), the differential receiver clock error is eliminated - the impact is at least below the noise level of the code observation of $1-2 \cdot 10^{-9} \cdot \tau^{-1}$.

The robot arm itself was calibrated with a LEICA laser tracker LTD 640 at the Geodetic Institute of Hannover (GIH). It was proven, that the positioning is accurate at the $0.25 \mathrm{~mm}$ level, [16].

\subsection{Mathematical Model of Group Delay Variations (GDV)}

In analogy to the approach for the determination of PCV, we use continuous expansions on a sphere for the suitable modelling of GDV as variations with orthogonal base-functions. The model reads:

$$
G D V(\alpha, e)=\sum_{n=0}^{n_{\max }} \sum_{m=0}^{m_{\max }}\left\{\begin{array}{l}
A_{n m} \bar{R}_{n m}(\alpha, e) \\
B_{n m} \bar{S}_{n m}(\alpha, e)
\end{array}\right\}
$$

with: $A_{n m}, B_{n m}:=$ unknown coefficients, and

$$
\left\{\begin{array}{c}
\bar{R}_{n m}(\alpha, z) \\
\bar{S}_{n m}(\alpha, z)
\end{array}\right\}=\left\{\begin{array}{c}
\cos (m \alpha) \\
\sin (m \alpha)
\end{array}\right\} N_{n m}\left(P_{n m} \sin (e)\right)
$$

the fully normalized harmonics $\bar{R}_{n m}$ and $\bar{S}_{n m}$, of degree $n$ and order $m$. These are functions of the elevation angle $e$ and azimuth angle $\alpha$ of a satellite in the antenna system. The 


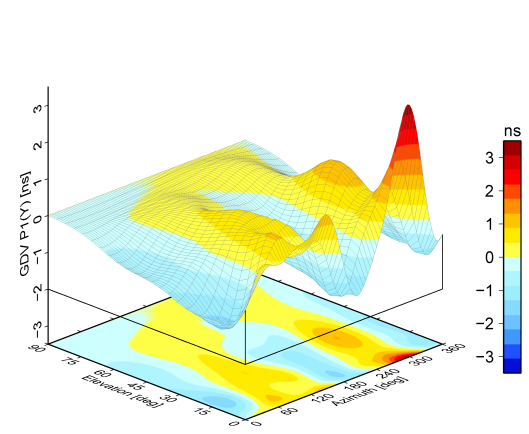

(a) ASHTECH P1

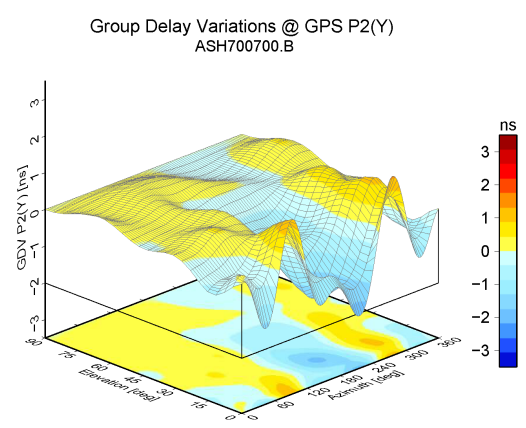

(d) ASHTECH P2

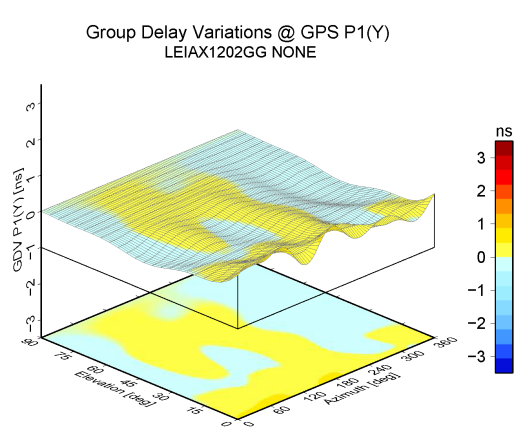

(b) LEIAX1202GG P1

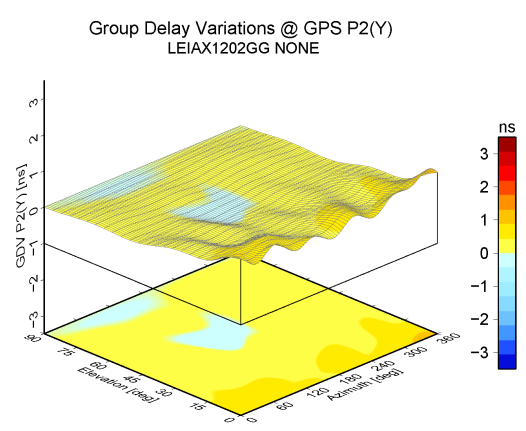

(e) LEIAX1202GG P2

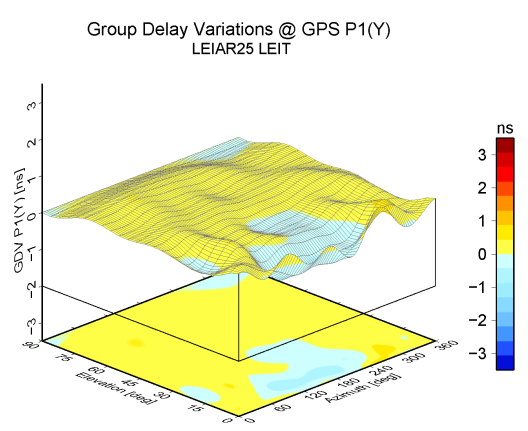

(c) LEIAR25 LEIT P1

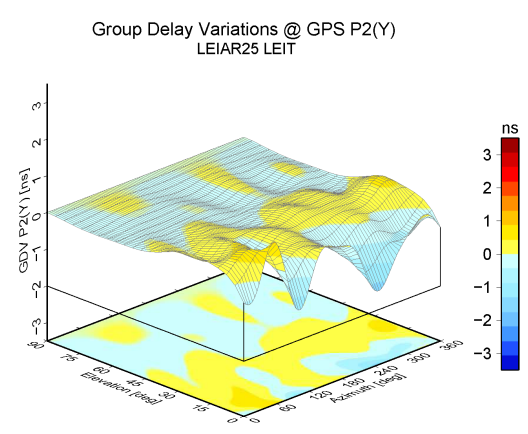

(f) LEIAR25 LEIT P2

Figure 2. Determined GDV for exemplary antennas.

normalization factor is denoted by $N_{n m}$ and the associated Legendre functions by $P_{n m}(\sin (e))$, [17].

The estimation procedure is implemented in the software package developed at the Institut für Erdmessung. Details as well as results of a co-variance analysis of the estimable parameters are described in [14] or [18]. The corrections were computed from a synthesis of the spherical harmonics.

\section{Discussion of Group Delay Variations (GDV)}

Applying the methodology, described above, we estimate parameter sets for different non-geodetic and geodetic antennae with different characteristics.

In Figure 2 the determined GDV are depicted for some exemplary antennae. The P1 and P2 GDV for an Ashtech Marine ASH700700B antenna are depicted in Figures 2(a) and 2(d). The plots show that elevation-dependent variations are present with magnitudes from $-2 \mathrm{~ns}<\mathrm{GDV}<+2.6 \mathrm{~ns}$, mainly caused by azimuth-dependent variations, cf. Figure 2(a). A similar GDV pattern was obtained for the P2 code, especially when comparing the P1 and P2 GDV at $300^{\circ}$ in azimuth. Azimuthal variations are smaller for $\mathrm{P} 2$ with magnitudes of approximate $-1.5 \mathrm{~ns}<\mathrm{GDV}<+1.5 \mathrm{~ns}$, cf. Figure 2(d).

The Figures 2(b) and 2(e) show the determined GDV of the Leica Rover LEIAX1202GG antenna which exhibits no significant elevation dependency, but small azimuthal variations at elevations below $15^{\circ}$. The behaviour on P1 is very similar to the GDV on P2, with magnitudes below $1 \mathrm{~ns}$, cf. Figure 2(e).

The GDV for the Leica choke ring LEIAR25 antenna, depicted in Figures 2(c) and 2(f) appear to be not elevationdependent. However, small azimuthal variations occur. Here the GDV show marginal differences between P1 and P2, apart from small variations at elevation angles below $15^{\circ}$.

For a calibrated $\mu$ Blox antenna azimuthal variations on C/A have magnitudes of up to 2-3 ns for elevation angles between $90^{\circ}-30^{\circ}$, cf. Figure 3(b). These azimuthal variations produce the overall effect, which can be seen in the single differences depicted in Figure 3(d) and 3(e). However, the GDV for the Javad Dual Depth choke ring antenna are very small and show only elevation dependencies below $1 \mathrm{~ns}$ at elevations below $15^{\circ}$, but not significant azimuthal variations as depicted in Figure 3(c).

Furthermore, some similarities of the GDV pattern exist with respect to their corresponding PCV pattern for the antennae used in this study. This is especially true for antennae, which show large azimuthal variations in their PCV like e.g. the Ashtech Marine ASH700700B or the $\mu$ Blox patch antenna.

\section{Plausibility checks}

Since the code noise is large compared to the pattern, the following plausibility checks for the determined GDV were carried out.

A short baseline set-up as depicted in Figure 3(a) was used to verify the obtained results. For this test, the cor- 


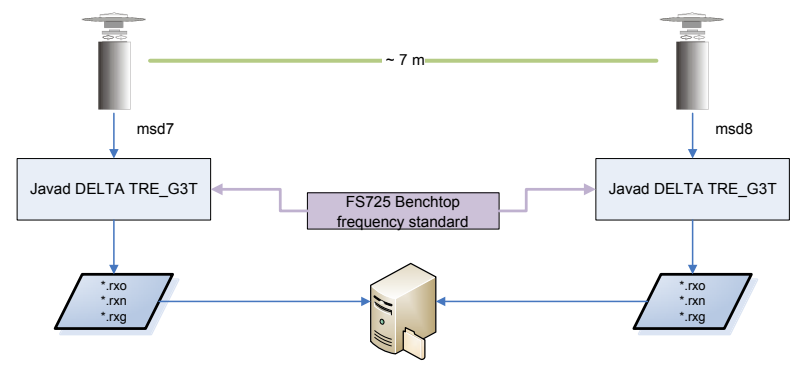

(a) short baseline setup

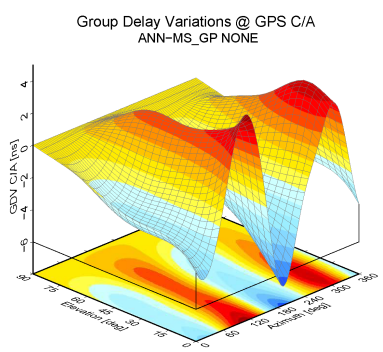

(b) GDV C/A UBLOX

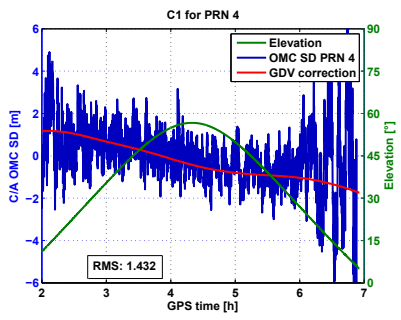

(d) SD on short baseline

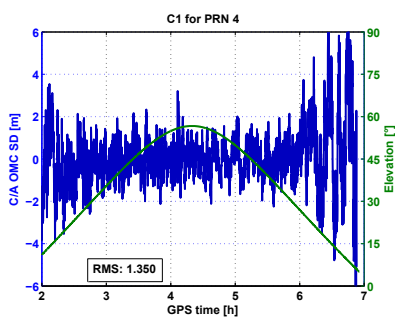

(f) GDV applied

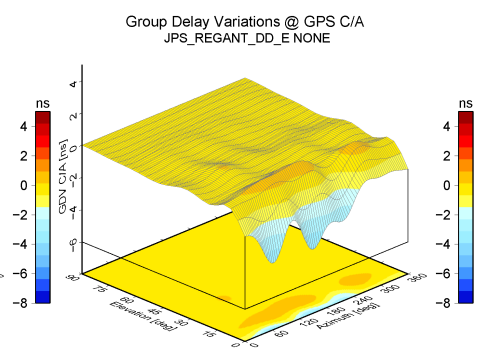

(c) GDV C/A JAVAD DualDepth

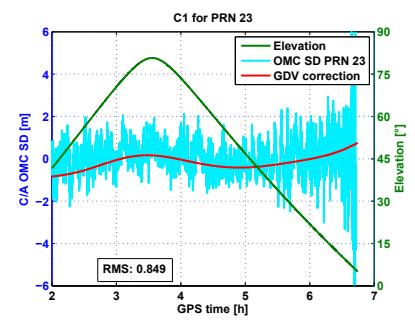

(e) SD on short baseline

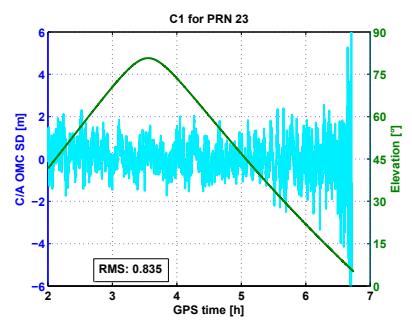

(g) GDV applied
Figure 3. (a) Short baseline common clock mode set up, (b) GDV of a $\mu$ Blox ANN-MS_GP, (c) GDV of a Javad Dual Depth JPS_REGANT_DD_E antenna, $(\mathrm{d}, \mathrm{e})$ inter-station SD for two exemplary satellites PRN4 and PRN23, (f,g) SD after applying GDV to the code observable.

rections per epoch and line-of-sight were calculated and applied to the observed minus computed values (OMC) of the inter-station single differences of the C/A code. A Javad Dual Depth JPS_REGANT_DD_E antenna was used on pillar MSD8 while a $\mu$ Blox ANN-MS_GP antenna with a ground plane was installed on pillar MSD7. The OMC of the C/A inter-station single differences (SD) are depicted in the Figures 3(d) and 3(e) versus GPS time. In addition the elevation of the satellites and the Group Delay Variations are indicated by a solid line. As shown in Figures 3(f) and 3(g) the rootmean-square (RMS) of the OMC C/A SD could be improved by up to $6 \%$ by applying the corrections.

Further tests were carried out using the same setup with a Trimble Zephyr I TRM41249.00 and a Leica AR25 LEIAR25 antenna mounted on pillars MSD7 and MD8, respectively. It turns out that applying the GDV for both antennae on a short baseline improves the code observation in the mean elevations about 0.6-0.8ns.

In a third evaluation, estimated GDV were applied to the observations as corrections during the calibration processing. Several calibrations were repeated, showing that GDV are significant and estimable, [18].

\section{Application to time transfer}

To analyze the impact of GDV on time and frequency transfer two scenarios were studied: (1) a simulation for continental and inter-continental time links, (2) a frequency transfer scenario using Precise Point Positioning (PPP) with code observations corrected for GDV.

\subsection{Simulation Strategy}

The GDV are introduced in a simulation. We processed 14 days in daily batches with a sampling rate of 15 seconds for 7 IGS (or ITRF) stations, (Table 1). Besides inner-European links stations outside Europe were used to study the impact of varying GPS satellite geometry. For each station we assume four scenarios with different antennae: (1) Ashtech Marine ASH700700.B, (2) Leica AR25 LEIAR25, (3) Trimble Zephyr I TRM41249.00 and (4) Trimble 2d choke ring TRM59900.00 antenna to evaluate the possible impact of different antenna combinations on different links. The receiver clocks are estimated using the ionosphere-free linear combination $P_{3}$,

$$
P_{3}=\left(\frac{f_{1}^{2}}{f_{1}^{2}-f_{2}^{2}}\right) \cdot P_{1}-\left(\frac{f_{2}^{2}}{f_{1}^{2}-f_{2}^{2}}\right) \cdot P_{2},
$$

with the carrier frequencies $f_{1}$ and $f_{2}$ and the corresponding code observations $P_{1}$ and $P_{2}$.

From experiments at the laboratory network at the IfE rooftop with different antennae and receivers a $P_{3}$ code noise of $\sigma_{P_{3}} \approx 0.6 \mathrm{~m}$ was obtained, as indicated by a slope of $\tau^{-1}$ in Figure 5(a).

Precise orbits from the IGS [19] as well as the software developed at IfE were used for the simulations. The applied time transfer methodology equals the All-In-View technique, since all satellites visible above an elevation of $5^{\circ}$ are considered. The advantage of using All-In - View w.r.t CommonView techniques are discussed in detail in [20]. For every station, receiver clock solutions with four antennae are obtained, each set with 80640 epochs with a 15 s sampling rate. Exemplary results for the receiver clock differences are depicted in Figure 4. Typical patterns are induced by the changing satellite geometry which repeats with the well known sidereal repetition time of $24 \mathrm{~h}-(3 \mathrm{~min} 56 \mathrm{sec})$. The induced time transfer error for the link PTBB-BRUS with the same antenna is 
Table 1. Stations used in the simulation scenario.

\begin{tabular}{lllr}
\hline Station & \multicolumn{1}{c}{ Location } & Institution & $\begin{array}{c}\text { Length } \\
{[\mathrm{km}]}\end{array}$ \\
\hline \hline PTBB & Braunschweig, Germany & PTB & 0 \\
\hline BRUS & Brüssel, Belgium & ROB & 479 \\
MIZU & Mizusawa, Japan & GFZ & 8785 \\
ONSA & Onsala, Sweden & LMV & 552 \\
UNSA & Salta, Argentinia & GFZ & 11242 \\
USN3 & Washington, USA & USNO & 6520 \\
WAB2 & Wabern, Switzerland & METAS & 679 \\
\hline
\end{tabular}

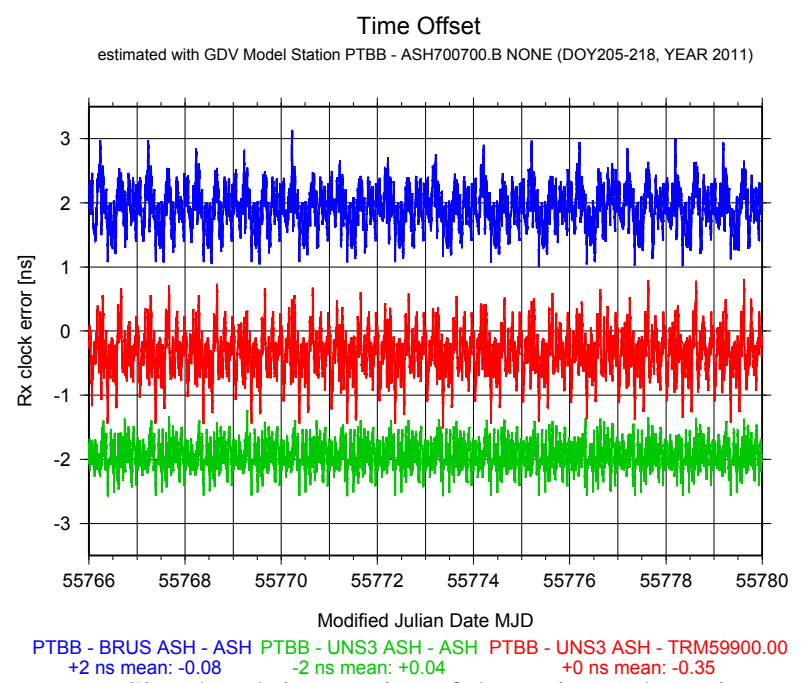

Figure 4. Simulated time series of the estimated receiver clock error for three exemplary links from PTBB are shown. Please note that the links PTBB-BRUS and PTBB UNS3 were shifted by $+2 \mathrm{~ns}$ and $-2 \mathrm{~ns}$, resp. for readability.

$+0.05 \mathrm{~ns}$ whereas for an inter-continental link PTBB - UNS3 equipped with the same antenna this increases to $-0.08 \mathrm{~ns}$. However, the largest impact is obtained for the inter-continental link PTBB - UNS3 and different antennae (ASH700700.B vs. TRM59900.00) with a time offset of $-0.35 \mathrm{~ns}$.

In Figure 5(a) the Allan Deviation [21] of several links, for simulation purposes equipped with same antenna type, a Trimble 2d choke ring (TRM59900.00) are shown. It is obvious that the Allan deviation starts with a white frequency modulation (WFM), with a characteristic slope of $\tau^{-0.5}$ until $\tau=10^{3} \sec (\approx 20$ minutes $)$. A noise of $\sigma_{P_{3}}=2 \cdot 10^{-9}$ at $\tau=$ $1 \mathrm{sec}$, is assumed, which is quite reasonable for the observation noise of $P_{3}$ links shown in [22] as well as [4]. It is obvious that the values of the Allan deviation for all kinds of links are definitely below the WPM of the code observation. In addition, the modified Allan deviation [23] depicted in Figure 5(b) shows, that the apparent noise induced by GDV behaves like WPM.

For a inner-European link PTBB - BRUS and for the same antenna, the plot of the Allan deviation shows a stability of $\sigma_{y}=3.5 \cdot 10^{-13}$ at $\tau=10^{3}$ seconds. For an inter-continental link PTBB - UNS3 this transition point changes to $\sigma_{y}=1.5$.

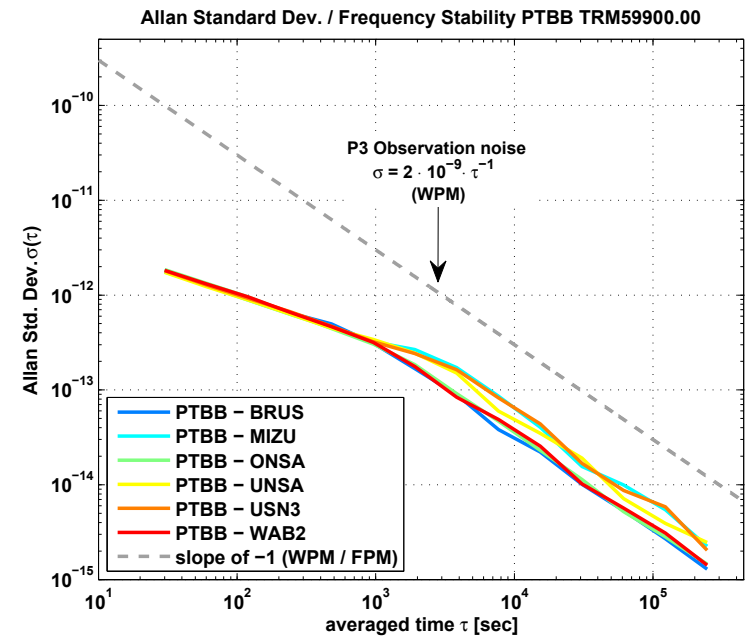

(a)

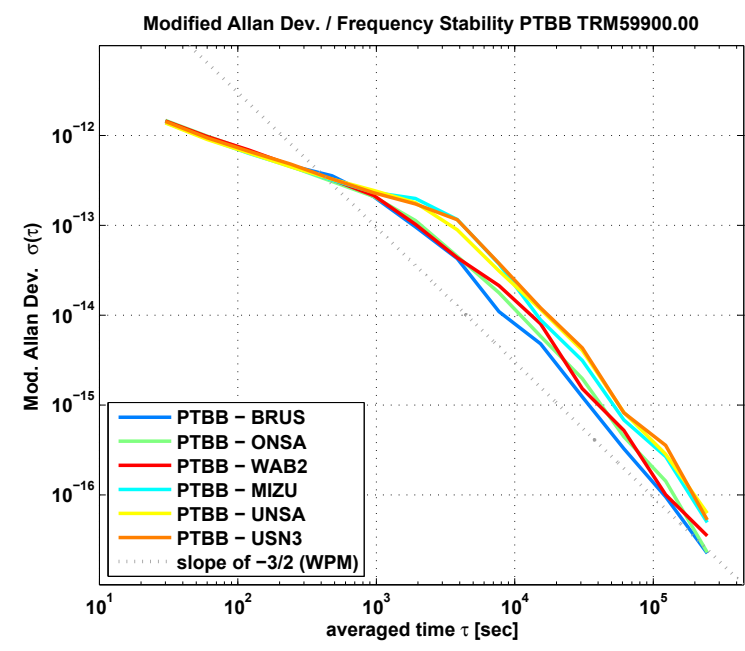

(b)

Figure 5. Allan standard deviation (a) and modified Allan deviation (b) of simulated time series for several baselines.

$10^{-13}$ at $\tau \approx 3.6 \cdot 10^{3}$ seconds. This shows that significant differences for larger $\tau$ occur due to the different GPS satellite geometry and variations accentuate for increasing distance of the link. The behaviour of the Allan deviation is very similar for all tested antennae, especially concerning the transition point. The transition between the different noise processes always occur around $\tau=10^{3}$ seconds for short links below $1000 \mathrm{~km}$, cf. Table 1 , and changes to $\tau=3.6 \cdot 10^{3}$ seconds for links of more than $6000 \mathrm{~km}$, cf. Figure $5(\mathrm{a})$. For all antenna combinations the impact is well below the $P_{3}$ observation noise of the links and thus has no impact on the stability.

To provide comparable values within the time and frequency transfer, the simulated time series were analyzed using the GPS time transfer standard GGTTS format. This format is a unique standard for GPS time receiver software and applications. One track of the GGTTS formats consists of 780 


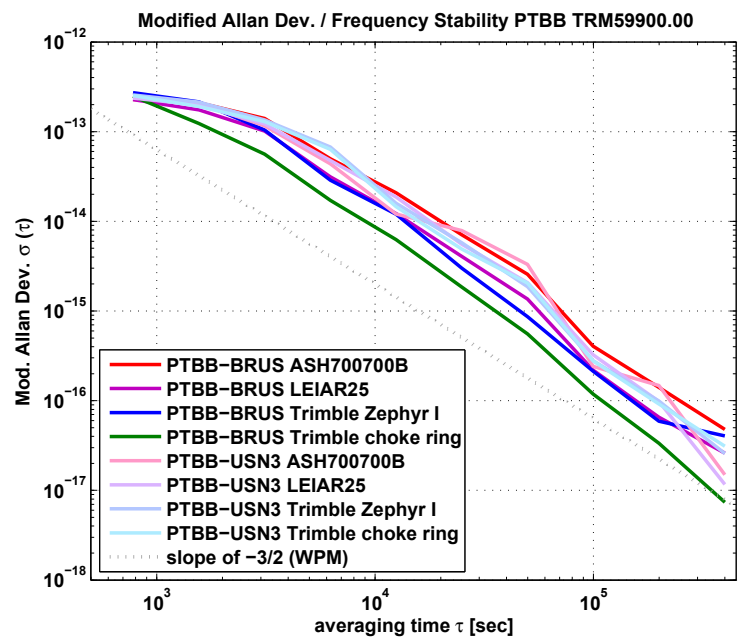

Figure 6. Modified Allan deviation of GGTTS formatted time series for several baselines and different equipment.

seconds (13 minutes) length. The reason for the 13 minutes are (1) usually a receiver required 2 minutes to lock on one satellite, (2) approx 10 minutes are necessary to transmit the full content of the navigation message and (3) one minute is usefully for data processing and preparation of a new track [24].

Simulated time series for several antennae and links are transformed into the GGTTS format and are depicted in Figure 6. For all antennae and links the apparent noise induced by the GDV is characterized by a slope of $\tau^{-3 / 2}$. As expected, the smallest mod. Allan deviation is obtained for the short link PTBB-BRUS, with the same antenna, since the impact of GDV is largely reduced because of the similar satellite geometry. The mod. Allan deviation [23] of the several links are very similar. For inter-continental links the mod. Allan deviation starts with a white frequency modulation (WFM) of $\sigma_{y}=5 \cdot 10^{-12} \tau^{-0.5}$, cf. also Figure 6 , followed by a white PM with $\sigma_{y}=2-6 \cdot 10^{-10} \tau^{-3 / 2}$ for $\tau>10^{3} \mathrm{sec}$. As described above, the mod. Allan deviation of the GGTTS time series is moreover well below the $P_{3}$ observation noise.

To summarize this subsection: the best results can be achieved assuming that both stations of a short link are equipped with the same antennae. For long links the impact depends on the GDV as well as on the changing satellite geometry. Within our analysis the GDV of antenna have a quite similar impact. Since the $P_{3}$ code noise is larger than the effect introduced by the GDV, they are not yet an issue for the stability of the link. The same holds for the GGTTS time format.

However the apparent noise induced by the GDV is close to the noise, achievable by combining carrier phase and code observables. The phase noise of the Code plus Carrier linear combination (CPC) is reduced by a factor of about 20 with respect to the $P_{3}$ code noise, [7]. Also potential GDV for the Galileo E5a,b AltBOC signal could have an impact on time transfer since this signal is more robust against external influences.

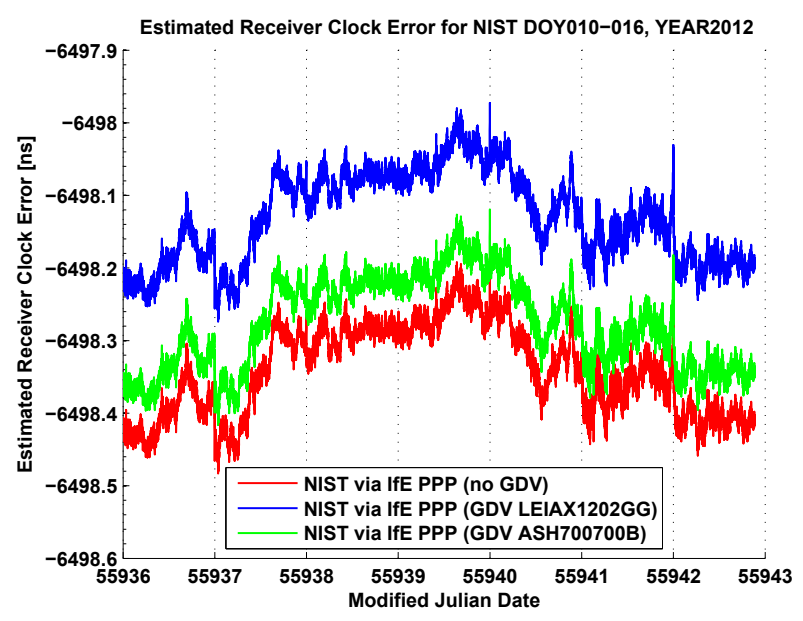

(a) Estimated receiver clock error at NIST

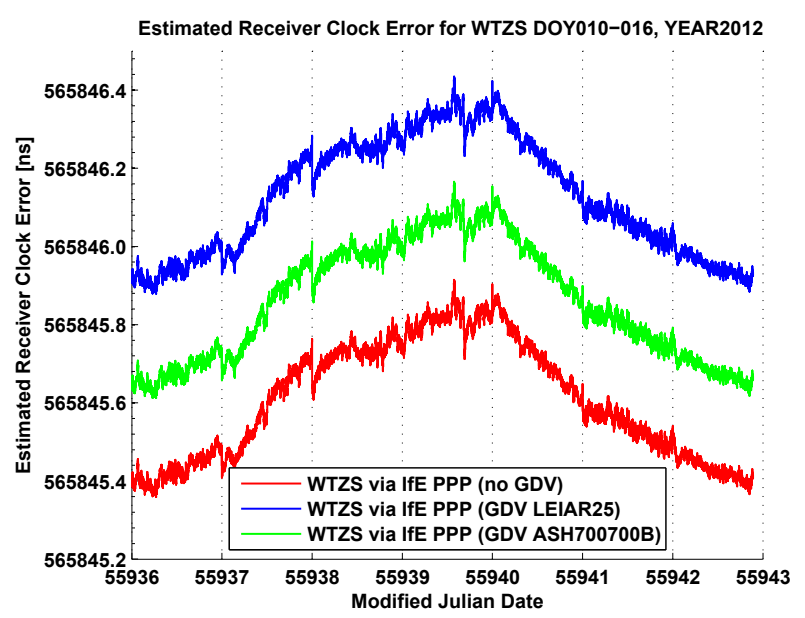

(b) Estimated receiver clock error at WTZS

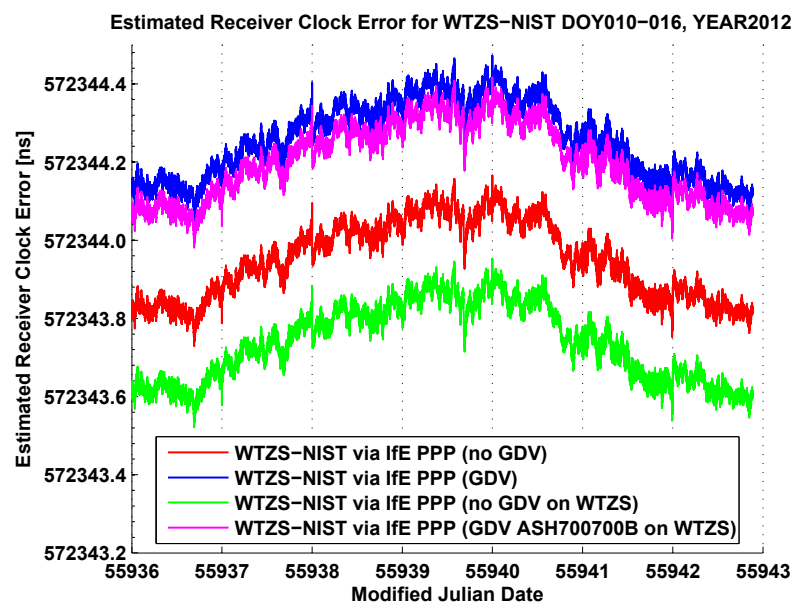

(c) Estimated receiver clock error for the link WTZS-NIST

Figure 7. PPP receiver clock estimates for station NIST and WTZS (linear drift removed) with different GDV applied. 


\subsection{PPP frequency transfer}

In order to study the impact of GDV on PPP frequency transfer, the GDV corrections were introduced into a Precise Point Positioning (PPP) link between Wettzell, Germany (WTZS) and Boulder, USA (NIST). This link is of special interest since (1) the GDV could be determined for the antennae, which are actually located at these stations, (2) the receivers at these stations are connected to active $\mathrm{H}$-Maser frequency standards, which provide the necessary frequency stability, and (3) from the simulation study the largest impact is expected for a intercontinental link.

The station WTZS is equipped with a Leica AR25.R3 LEIT 3d choke ring antenna and a Septentrio PolaRx2 receiver connected to an external H-Maser EFOS 39. The station NIST uses a Novatel NOV702 antenna in combination with a Novatel NOV OEM4-G2 receiver also connected to an external H-Maser. In our study, a LEIAX1202GG is used for NIST instead of a NOV702 but the antennae are nearly identical.

The PPP analysis is carried out with a PPP software, developed at IfE [25] using a Kalman filter approach. The precise orbits, clocks, and Earth rotation parameter (ERP) for the DOY010 up to DOY016, 2012 were obtained from the IGS, [19]; corrections for ocean tide loading (Model FES2004) origin from the Onsala Space Observatory (OSO), cf. [26].

For this study, several scenarios were calculated: (1) only one day is processed in order to analyse the impact on a daily solution, (2) one week of data were processed continuously and (3) in both scenarios a third antenna, Ashtech Marine ASH700700B which has a more pronounced GDV pattern than the LEIAR25.R3 and LEIAX1202GG antennae is introduced.

Figures 7(a) and 7(b) show the receiver clock error calculated for both stations separately. The three time series are obtained from (1) applying no GDV correction, (2) applying the GDV correction corresponding to the antenna in use, and (3) applying arbitrarily the correction for an Ashtech Marine antenna. A common linear drift is removed. It can be noted that the time series of the receiver clock estimates of station NIST are nearly identical except to an offset of $0.2 \mathrm{~ns}$ between with and without considering the GDV. The same behavior is depicted for the estimated receiver clock of station WTZS, where an offset of up to $0.5 \mathrm{~ns}$ can be noticed. As shown in Figure 7(c) the impact of the GDV corrected code observable introduce an offset with a magnitude of $0.35 \mathrm{~ns}$ on the inter-continental link WTZS - NIST. Additionally, we have introduced a second antenna correction (Ashtech Marine ASH700700.B) to see the impact of a more pronounced GDV pattern. In conclusion, the difference between the solutions with and without consideration of GDV introduces an offset, but the patterns of the receiver clock estimates are very similar, cf. Figures 7(a) -7(c).

Concerning the frequency stability of the link, we compare two scenarios. First we start with a daily solution, the

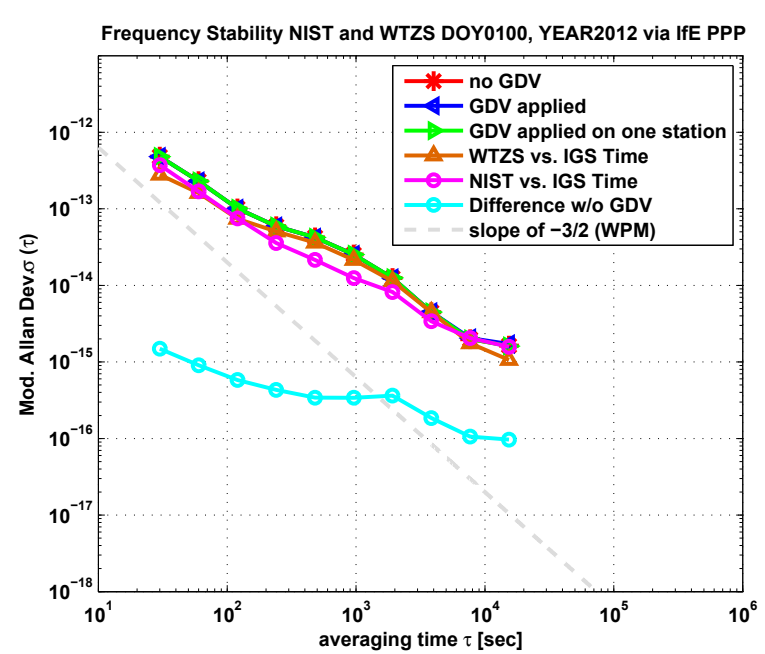

(a)

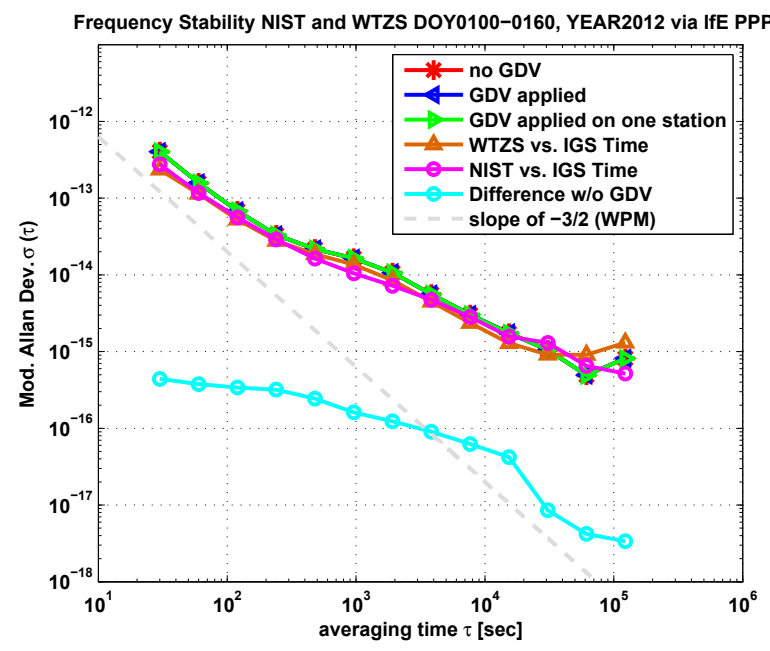

(b)

Figure 8. Frequency Stability for intercontinental time link WTZS and NIST for one day (a) and one week (b). Data, origin from daily IGS station files, processed with IfE PPP software to analyze the impact of GDVs.

modified Allan deviation for different scenarios is depicted in Figure 8(a). The stability for one day resembles a flicker phase modulation (FPM) process of $1.5 \cdot 10^{-12} \cdot \tau^{-1}$ for the whole day. Significant differences between the several PPP processing scenarios could not be detected. But if we take a look onto the stability of the difference between w/o GDV consideration, one summarize, that the process is mapped as a white frequency modulation (WFM) with $3.5 \cdot 10^{-15} \cdot \tau^{-0.5}$.

In the second scenario, data of one week from the PPP link WTZS-NIST is depicted in Figure 8(b). The modified Allan deviation starts with a white phase modulation (WPM) and transitions into a flicker phase modulation (FPM) process with $5 \cdot 10^{-13} \cdot \tau^{-1}$ down to $8.46 \cdot 10^{4} \mathrm{sec}$ (one day). For $\tau>$ $8.46 \cdot 10^{4}$ seconds the frequency stability turns into a flicker 
frequency modulation (FFM) process with a magnitude of about $8-9 \cdot 10^{-16} \cdot \tau^{0}$, which is typical for PPP frequency transfer, since this level of FFM process reflects the frequency stability of the used H-Masers.

To sum up, in currently used PPP links GDV leads to offsets of few tens of nanoseconds. However, GDV are not an issue for the stability of the link since the code weighting with $\sigma_{P_{3}}=0.6 \mathrm{~m}$ down-weights the impact of the P3 GDV magnitudes when using geodetic antennae.

\section{Conclusion}

In this study we have shown that significant systematic errors on code observables can be caused by Group Delay Variations (GDV). GDV vary with the elevation as well as the azimuth of the incident ray and are specific for each antenna type. Furthermore it could be shown that GDV can be estimated by the Hannover Concept of absolute antenna calibration. The method provides a precision of $0.15 \mathrm{~ns}$. Furthermore, it could be shown that the RMS of the code OMC could be reduced by up to $6 \%$ when applying these corrections.

Simulation studies reveal that the effect of GDV equals a white PM / white FM and a stability of $\sigma_{y}=3.5 \cdot 10^{-13}$ at $\tau=10^{3}$ seconds. The same behaviour is obtained when applying the GGTTS standard format for time and frequency transfer. It could be shown, that the GDV introduce a white PM on the GGTTS formatted time series. Since the noise level of P3 is rather high $\left(\sigma_{P_{3}} \approx 2 \cdot 10^{-12}\right.$ at $\left.\tau=10^{3} \mathrm{sec}\right)$, the impact of the GDV on the stability of P3 time and frequency links can be neglected up to now. However, GDV can induce time offsets in the links of up to $0.5 \mathrm{~ns}$.

Furthermore the introduction of GDV in a PPP frequency comparison on an inter-continental link between Wettzell WTZS and Boulder NIST shows, that GDV are not a limiting factor for the stablility of the link, however offsets of up to $0.4 \mathrm{~ns}$ can be introduced.

Finally, further investigations are necessary to analyse the impact of GDV on code and carrier phase combinations. Here GDV may become an issue because of the new generations of code modulation, such as Galileo E5 AltBOC, which provides a very low code noise. This new signals will be fully available within the next years and improve time and frequency transfer as well. Therefore GDV can become an issue, so that they have to be modelled accurately for the use in precise applications.

\section{Disclaimer}

Although the authors dispense with endorsement of any of the products used in this study, commercial products are named for scientific transparency. Please note that a different receiver / antenna unit of the same manufacturer and type may show different characteristics.

\section{Acknowledgment}

The work within this project is supported by the German Aerospace Center (DLR) with the project label 50NA0903, funded by the Federal Ministry of Economics and Technology (BMWI), based on a resolution by the German Bundestag.

\section{References}

[1] Al Gifford. One Way GPS Time Transfer 2000. In 32nd Annual Precise Time and Time Interval (PTTI) Meeting, 2000.

[2] J. Levine. A Review of Time and Frequency Transfer Methods. Metrologia, 45:162-174, 2008.

[3] Michael A. Lombardi, Lisa M. Nelson, Andrew N. Novick, and Victor S. Zhang. Time and Frequency Measurements Using the Global Positioning Systems. Cal. Lab. Int. J. Metrology, 3:26-33, 2001.

[4] Hsin-Min Peng, Chia-Shu Liao, and Jeng-Kuang Hwang. Perfomance Testing of Time Comparison Using GPSsmoothed P3 Code and IGS Ephemeries. IEEE Transactions on Instrumentation and Measurement, 54(2):825828, 2005.

[5] Tim Murphy, Preston Geren, and Tom Pankaskie. GPS Antenna Group Delay Variation Induced Errors in a GNSS Based Precision Approach and Landing Systems. In Proc. ION GNSS 20th International Technical Meeting of the Satellite Division, 25-28 September, Fort Worth, Texas, 2007.

[6] Bruce Haines, Yoaz Bar-Sever, Willy Bertiger, Shailen Desai, Nate Harvey, and Jan Weiss. Improved Models of the GPS Satellite Antenna Phase and Group Delay Variations Using Data from Low-Earth Orbiters, Abstract \#G54A-05. In presented at 2010 Fall Meeting, AGU, San Francisco, Calif., 13-17 Dec, 2010.

[7] Pascale Defraigne. GNSS Time and Frequency Transfer: state of the art and possible evolution. In Workshop on Development of Advanced TFT Techniques, Bureau Internationale des Poids et Measurements (BIPM), June 28, 2011.

[8] Giancarlo Cerretto, Andrea Perucca, Patrizia Pavella, Avaro Mozo, Ricardo Píriz, and Miguel Romay. Network Time and Frequency Transfer With GNSS Receivers Located in Time Laboratories. IEEE Transactions on Ultrasonics, Ferroelectronics and Frequency Control, 57(6):1276-1284, 2010.

[9] Jim Ray and Ken Senior. IGS/BIPM pilot project: GPS carrier phase for time/frequency transfer and timescale formation. Metrologica, 40:270-288, 2003.

[10] Gerhard Wübbena, Martin Schmitz, and M. Propp. Antenna Group Delay Calibration with the Geo++ Robot extensions to code observable. In IGS Analysis Workshop, Poster, June 2-6 Miami Beach Florida, USA, 2008. 
[11] Falko Menge, Guenter Seeber, Christian Völksen, Gerhard Wübbena, and Martin Schmitz. Results of the absolute field calibration of GPS antenna PCV. In Proc. Int. Tech. Meet ION GPS-98, Nashville Tennesee, 1998.

[12] Gerhard Wübbena, Martin Schmitz, Falko Menge, Volker Böder, and Guenter Seeber. Automated Absolute Field Calibration of GPS Antennas in Real-Time. In ION GPS 2000, Salt Lake City, 19-22. September 2000, USA, 2000.

[13] Falko Menge. Zur Kalibrierung der Phasenzentrumsvariationen von GPS Antennen für die hochpräziese Positionsbestimmung. PhD thesis, Wisschschaftliche Arbeiten der Fachrichtung Vermessungswesen der Universität Hannover, 2003.

[14] Tobias Kersten and Steffen Schön. Towards Modeling Phase Center Variations for Multi-Frequency and MultiGNSS. In 5th ESA Workshop on Satellite Navigation Technologies and European Workshop on GNSS Signals and Signal Processing (NAVITEC), 8.-10. December ESTEC, Noordwijk, The Netherlands December, 2010.

[15] Günter Seeber. Satellite Geodesy, Foundations, Methods and Applications. Walter de Gruyter, 2003.

[16] Vincent Meiser. Kalibrierung des GNSSAntennenkalibrierroboters des Institut für Erdmessung mittels Lasertracking. Technical report, Geodätisches Institut Hannover, 2009.

[17] E.W. Hobson. The Theory of Spherical and Ellipsoidal Harmonics. Cambridge, University Press, 1931.

[18] Tobias Kersten and Steffen Schön. GNSS Group Delay Variations - Potential for improving GNSS based Time and Frequency Transfer? In Proceedings of the 43rd Annual Precise Time and Time Interval (PTTI) Meeting, Long Beach, CA, USA, 2011.

[19] John M. Dow, R.E. Neilan, and Chris Rizos. The International GNSS Service in a changing Landscape of Global Navigation Satellite Systems. Journal of Geodesy, Springer Verlag, New York, 83:191-198, 2009.

[20] Marc A. Weiss, Gerard Petit, and Z. Jiang. A Comparison of GPS Common-View Time Transfer to All-in-View. In Frequency Control Symposium and Exposition, 2005. Proceedings of the 2005 IEEE International. 29-31 Aug., 2005.

[21] David W. Allan. Statistics of Atomic Frequency Standards. Proceedings of the IEEE, 54(2):221-230, 1966.

[22] Pascale Defraigne and G. Petit. Time transfer to TAI using geodetic receivers. Metrologia, 40:184-188, 2003.

[23] L. G. Bernier. Theoretical Analysis of the Modified Allan Variance. In 41st Annual Frequency Control Symposium, pages 116-121, 1987.

[24] David W. Allan and Claudine Thomas. Technical Directives for Standardization of GPS Time Receiver Software to be implemented for improving the accuracy of GPS common-view time transfer. Metrologia, 31:69-79, 1994.
[25] Ulrich Weinbach and Steffen Schön. GNSS receiver clock modeling when using high Precision oscillators and its impact on PPP. Advances in Space Research, 47(2):229$238,2011$.

[26] H.-G. Scherneck. A parameterized Solid Earth Tide Model and Ocean Tide Loading Effects for global geodetic baseline measurements. Geophys. J. Int., 106(3):677-694, 1991. 